\title{
LA COMPLEXITE AFRO-COLOMBIENNE
}

\author{
Angélica Montes Montoya ${ }^{1}$
}

Le discours paradigmatique du multiculturalisme politique $e^{2}$, nourri par les études culturelles, des études subalternes, des théories anthropologiques, de la postcolonialité et de la postmodernité, a traversé les différentes sphères de la société colombienne (culturelles, politiques et socio-économiques) jusqu'à générer un discours qui s'installe dans l'ordre du « sens commun » et qui s'enferme parfois et évite le débat.

Ainsi que le signale Eduardo Restrepo, en se référant aux spécialistes du sujet de l'identité noire en Colombie, cette « communauté d'argumentation » produit un nombre considérable de textes qui, bien qu'ils appellent à dénoncer la marginalité de la population noire colombienne, laissent généralement de côté la théorisation des dilemmes que comporte le fait d'aborder le sujet de la pluralité culturelle et de sa reconnaissance:

$1 \quad$ PhD. en Filosofía Política de la Universidad Paris 8, Magíster en Estudio de Sociedades latinoamericanas de la Universidad Sorbona Nouvelle (Paris 3); titular de un diploma de Filosofa de la Universidad de Cartagena y de una Especialización en Ética y política de la misma universidad. Actualmente se desempeña como docente del ESSEC (Escuela Superior de Ciencias Económicas y Comerciales) y en la Universidad Paris 13 en Francia. Ha sido profesora invitada en varias universidades colombianas y mexicanas. Sus trabajos giran en torno al multiculturalismo político, la "différence", la relación entre memoria e historia, la "créolisacion" y el cosmopolitismo. Dirige el GRECOL-ALC (Grupo de reflexiones y estudios sobre Colombia y América Latina, asociado a la FMSH).

2 La définition politique la plus fédératrice du multiculturalisme y voit « les stratégies et les lignes adoptées pour ordonner ou régir la diversité et la multiplicité qu'engendrent les sociétés multiculturelles », c'est-à-dire qu'il constitue une stratégie d'administration politique des complexités, conflits, défis, problèmes de gouvernance entraînés par le fait de la pluriculturalité ou de la multiculturalité. 
Le dilemme comporte plusieurs facettes. La plus évidente est d'imaginer une conceptualisation et une politique de la différence et de l'ethnicité en ouvrant les notions du culturel, du territorial et de l'identitaire pour rendre compte de la multiplicité et de la complexité des pratiques, des relations et des représentations tissées autour et par les gens noirs dans le pays. Une autre facette moins évidente se rapporte à théoriser et à intervenir non seulement depuis la supposition du caractère progressiste et d'empowerment des reconnaissances dans le cadre des politiques du multiculturalisme d'État, mais d'examiner en même temps les risques, les défis et les fixations que précisément ces reconnaissances ont apportées et comment peuvent s'imaginer de nouvelles ruptures et de nouveaux schémas (Restrepo, 2005, p.21).

En effet, les discours autour de l'identité noire " afro $»^{3}$ de la Colombie, ont agi comme le chant des sirènes qui séduisent les marins dans l'Odyssée. Les spécialistes en sciences sociales (sociologues, anthropologues, politologues et historiens) se sont attachés à énumérer les aspects positifs du tournant pluraliste culturel intervenu avec la Constitution Politique de 1991, la dette historique de l'État envers les populations noires de ce pays, mais nous cherchons ici à théoriser les effets non positifs que ce changement peut générer ou génère dans les mentalités et les imaginaires sociaux ${ }^{4}$ (Taylor, 2004) de la société colombienne.

3 Ainsi que l'explique Restrepo, la catégorie apparaît dans les années 1950 dans le cadre d'un désir de montrer, depuis l'anthropologie et l'histoire, la particularité du Noir des Amériques. Lintention était d'établir les permanences et les changements culturels des populations noires à l'égard de l'Afrique, en établissant de cette façon une continuité historique : "La catégorie d'afro-colombien était l'adaptation au contexte colombien du concept d'afro-américain élaboré initialement aux Etats-Unis (où le terme " américain " est fréquemment confondu avec "étatsunien »). Avec cette notion on visait à insister sur l'héritage africain comme critère de spécificité qui marquait les "cultures noires "sur le continent américain ». Parmi les auteurs qui représentent ce premier moment de la théorie, dans les années 1950 et 1960, figurent Achille Escalante ou Raphaël Arboleda. Ce premier moment sera suivi, dans les années 1970, les points de vue fonctionnaliste, marxiste et l'écologie culturelle nord-américaine, et, plus récemment, le structuralisme français, l'interprétativisme nord-américain et les études raciales et identitaires de l'anthropologie sociale anglaise (Restrepo, 2005, p. 37).

4 Cette notion est proche de l'idée de « représentations sociales » travaillée par Moscovici (1989), Berger et Luckmann (1991), que nous pourrions résumer ainsi « Comme systèmes de 
Peut-être l'une des raisons de ce « silence » ou de cette « omission »» est que s'interroger sur les conséquences depuis un point de vue normatif (théorique) n'entre pas dans l'orbite de leurs intérêts disciplinaires, alors que cet aspect, au contraire, intéresse la philosophie sociale et politique, qui, soit dit en passant, est la grande absente du débat autour de ce sujet spécifique. Pourquoi disons-nous que la question des possibles « effets pervers » d'une politique de la reconnaissance de l'« afro » en Colombie, est un sujet qui n'est pas abordé avec le même confort?

Nous pouvons citer, comme élément de réponse, quelques considérations d'ordre sociologique, à savoir le ton passionné et politisé (dans le sens que donne Rancière à la politique) sur lequel est abordé le sujet depuis une décennie. Nous savons déjà que le Noir comme sujet de l'histoire - politique, culturelle et sociale - a été l'objet d'une « invisibilisation », sur laquelle on commence à s'interroger, progressivement, à partir des années 1980. Durant cette période, les travaux historiographiques, anthropologiques, ethnographiques et sociologiques de « révision » du discours dominant prennent de l'importance (Restrepo, 2005) et viennent s'associer aux mouvements sociaux de la fin des années 1980, porteurs, eux-mêmes, du discours des droits, de la démocratie participative et du renforcement de la citoyenneté et de la société civile.

codes, de valeurs, de logiques classificatoires, de principes interprétatifs et orientant les pratiques, qui définissent ce que l'on appelle conscience collective, qui s'érige avec une force normative en tant qu'elle institue les limites et les possibilités de la façon dont les femmes et les hommes agissent dans le monde " (Araya, 2002, p. 11). Cependant, la manière dont nous interprétons les « imaginaires sociaux» dans le présent texte s'éloigne de cette approche, étant donné que les « imaginaires sociaux », comme le propose Taylor (2004), à partir du regard philosophique, portent une attention particulière aux substrats ou aux horizons de valeurs qui donnent un sens aux pratiques sociales d'une communauté, alors que pour les théoriciens des représentations, l'important est dans l'interaction des sujets dans le cadre de ces substrats ou horizons, et ce parce que « ce qui permet de qualifier les représentations sociales est moins leurs supports individuels ou de groupes que le fait qu'elles soient élaborées au cours du processus d'échanges et d'interactions» (Araya , 2000). 
Dans ce contexte, l'« essentialisme stratégique » (Spivak, 1987), consistant à appliquer un discours opérationnel de l'identité ethnoculturelle, a été une tactique employée à partir des années 1990 et qui participe du processus de l'inclusion de la question de l'identité culturelle de la population noire, sur la scène académique, sociale et politique de la Colombie. En ce sens, nous pouvons affirmer que l'« afro-colombianité » constitue l'« identité ethno-raciale opérationnelle » qui a rendu possible la représentation politique de la condition du Noir dans ce pays. De cette façon s'est mise en marche l'ethnisation de la « communauté noire » du Pacifique colombien, dont parle la loi 70 de 1993, ainsi:

[On] suppose que le sujet politique et de droits retombe sur la notion d'un groupe ethnique avec une culture propre et différente de l'ensemble de la société colombienne. Cette culture propre serait le résultat de la descendance commune qui remonte à l'Afrique et aux expériences d'esclavagisation et de résistance partagées, mais surtout au développement de formes d'organisation et de pratiques traditionnelles de production de « communautés » sur quelques territoires spécifiques qui mettraient en évidence une logique culturelle particulière garante de la conservation de l'environnement (Restrepo, 2007, p. 476).

Ceci implique que l'ethnie est interprétée comme l'ensemble de caractéristiques, essentielles ou primordiales, propres à un groupe, qui reste, grâce à elle, défini dans sa différence face au reste de la société. C'est-à-dire que l'ethnie ne doit pas être considérée comme une simple construction $^{5}$, toujours mobile et en négociation, mais elle doit être vue comme profilant et légitimant l'« intervention politique spécifique dans les sphères locales, nationales et transnationales au nom de la demande d'une série

5 Comme l'explique Restrepo (2005, p. 117), deux positions clairement différenciées à propos de la notion d'ethnie coexistent : celle du « sud» décrite quelques lignes plus haut et celle $\mathrm{du}$ «nord » (anglophone) qui, depuis une posture constructiviste, observe l'ethnie comme contingente, comme une construction historique. 
de droitséconomiques et culturels dérivés de sa condition ethnique 》 (Restrepo, 2005, p. 217). Avec cette situation, on court le risque de présenter les sujets noirs « afro-colombiens » comme des sujets essentialisés ethnicoracialement et ontologisés (fondés ontologiquement).

Malgré le fait que l'identité ethno-raciale « opérationnelle » ait obtenu de bons résultats, en permettant à la population noire colombienne de prendre conscience de sa propre condition de marginalité et en provoquant, chez les leaders et les représentants des populations noires colombiennes, une résistance fondée sur " des solidarités temporelles tournées vers l'action sociale, au moyen desquelles ils acceptent de manière transitoire une position essentialiste quant à leurs identités pour pouvoir agir comme un bloc " (Mosquera, 2007, p. 214), elle ne devrait cependant pas durer très longtemps comme stratégie théorique, c'est-à-dire comme façon de justifier la nécessité et la pertinence d'aborder, de promouvoir et d'entretenir la discussion autour de la pluralité culturelle en général.

Ainsi, il est légitime de se demander : sous quelles conditions cette « identité opérationnelle afro » est-elle vecteur de nouvelles exclusions ou de tensions ? Un usage exagéré de l'« essentialisme stratégique » ne conduit-il pas aux mêmes défauts et aux mêmes vices que l'essentialisme traditionnel, pratiqué historiquement par les groupes hégémoniques ? Comment être sûrs que l'identité stratégique "afro » ne finira pas par enfermer dans la notion d'« afro » toutes les expériences possibles des populations noires? En d'autres termes : comment empêcher que l'usage stratégique ne finisse par s'imposer comme nouvel impératif d'interprétation, en normativisant et en fixant l'identité ?

Nous ne cherchons pas à suggérer que les points de vue de l'« afro-américanité » (qui soulignent les legs historiques et culturels africains) seraient erronés, inutiles ou qu'ils devraient être abandonnés, mais, plutôt, nous souhaitons mettre en exergue les « angles morts » de ce discours. C'est pour cette raison que nous partageons avec 
l'anthropologue colombien Eduardo Restrepo l'idée de la nécessité de mettre en question, d'abord, le modèle conceptuel et explicatif, et, ensuite, la stratégie narrative, qui sont à la base du discours de l'« afrocolombianité » (Restrepo, 2005, p. 78).

Eduardo Restrepo rapporte lui-même les arguments qui, depuis des décennies, ont été avancés pour atténuer la force explicative de la catégorie " afro-américanité », basée sur une "trace d'africanie », qui met en avant l'« afro-genèse $»^{6}$ en tant qu'élément annulé par l« eurogenèse» dominante. Selon Restrepo, l'inconvénient de parler d'une « trace d'africanie » (Friedemann) réside en ce que cela suppose de penser la culture africaine comme une unité et une totalité. C'est pourquoi il convient de parler, avant d'évoquer des « formes socioculturelles observables », de principes «grammaticaux» inconscients ou d'« orientations cognitives » partagées : des sortes de valeurs qui orientent les relations entre les individus et qu'il serait possible de retracer dans l'histoire ${ }^{7}$. Ceci permet de supposer que les Africains (d'Afrique Centrale et de l'Ouest) arrivés aux Amériques ne sont pas arrivés avec une culture déjà définie et qui aurait été transmise d'une génération à l'autre

6 L' "afro-genèse", met l'accent sur la participation des complexes culturels d'origine africaine à la construction des cultures et des sociétés latino-américaines. Son opposé serait l' "euro-genèse".

7 Depuis le Structuralisme de Lévi-Strauss, ces éléments "grammaticaux" ou "cognitifs" inconscients pourraient être partagés par divers groupes, en divers lieux de la planète, au-delà de la filiation raciale ou ethnique. Les travaux de Lévi-Strauss à propos du Totémisme et du mythe offrent des pistes qui peuvent faire douter de cette argumentation. Sa célèbre conférence à l'UNESCO, "Race et Histoire" (1952) illustre son effort pour montrer, depuis le structuralisme, la richesse que représente la diversité de cultures et combien il est inapproprié de s'enfermer dans une vision « ethnocentrique » de celle-ci à partir des notions de civilisation ou de progrès. Limpact de cette conférence, qualifiée par certains de manifeste antiraciste, sera amplifié par la deuxième conférence à l'UNESCO, "Race et culture" (1971). Celle-ci a connu une réception " scandaleuse », étant perçue comme une négation de la première, étant donné l'accent qui y était mis sur le caractère conflictuel de la relation entre les populations de cultures différentes qui partagent un même espace géographique, dont le contact prolongé ne contribue qu'à l' "uniformisation et l'appauvrissement des répertoires culturels respectifs" (Hernández, 2009, p. 90), situation qui met en danger la diversité culturelle appréciée et défendue par l'auteur. 

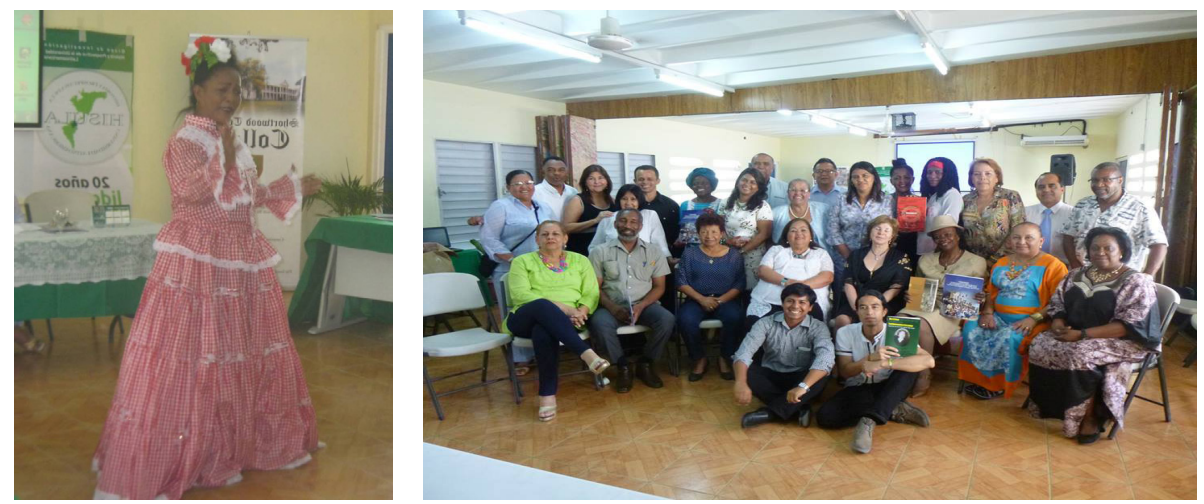

I Coloquio "La Historia de las Maestras Africanas y Afrodescendientes en Colombia, Jamaica, Brasil, Guatemala, Guinea Ecuatorial, Venezuela, Siglo Xx A Xxi”. Kingston, Jamaica, mayo 5-7 De 2015

jusqu'à nos jours, cette culture se construit ou se forme une fois les

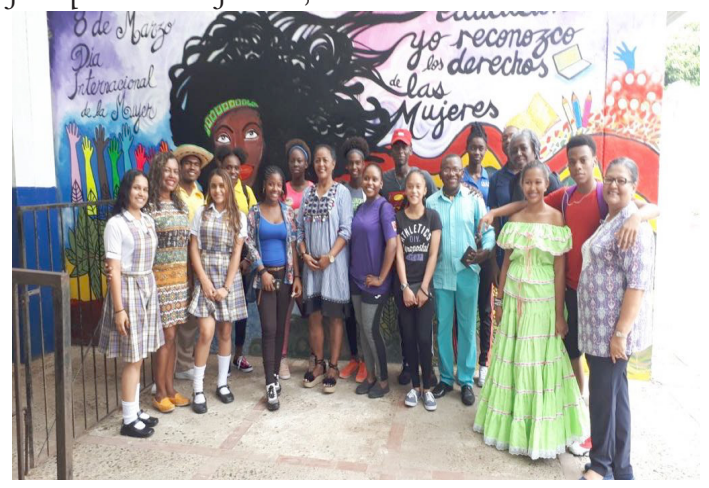

"Visita a la IE Antonia Santos, de docentes y estudiantes del Colegio Diego Martin Central School, de Trinidad \& Tobago, quienes se encontraban en Cartagena en un intercambio gestionado por el Embajador de Colombia en Trinidad \& Tobago, Dr. Alfonso Múnera Cavadia, con el apoyo de la Cámara de Comercio de Cartagena y la Universidad de Cartagena, dando inicio al intercambio de instituciones afro".

individus installés sur le Conmemoración muerte del cantante Joe Arroyo en la Institución Etnoeducativa Antonia Santos, sede San Luis Gonzaga

nouveau territoire et selon les nouveaux contextes ${ }^{8}$.

8 Mintz et Price, cités par Restrepo, 2005, p. 82.

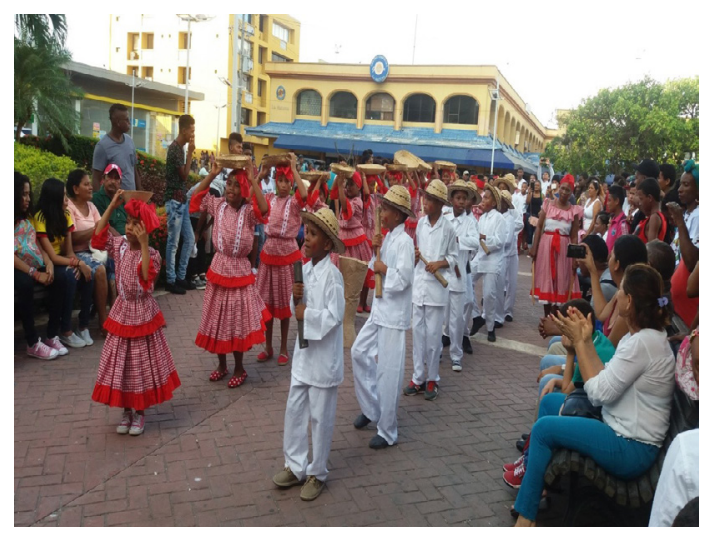


Pour se défendre de cette attaque, certains auteurs affirment qu'il s'agit là d'africanie et non d'africanité : la première renvoie à une mémoire construite à partir des souvenirs d'africanitég. En d'autres termes, africanité désigne le fait d'être africain, alors qu'africanie désigne la manière de se penser dans une relation ancestrale avec l'Afrique Noire, une mémoire de résistance. De même, ils soutiennent qu'il existe une « épistémologie » locale, propre à l'« afro », et qu'elle correspondrait aux principes « grammaticaux »» inconscients que citent les premiers critiques du discours de l'« afro-américanité ».

En Colombie, cette posture est assumée et défendue à partir d'un cas cité, travaillé et exhibé comme preuve vivante : le Palenque de San Basilio $^{10}$. Il faut préciser qu'en défendant cette position, on omettrait le fait que l'esclavage n'a pas seulement été une entreprise mercantile (Quijano, 2000), mais qu'il a entraîné, aussi, des processus de reconfiguration d'identités, culturelles, sociales, etc. Il est donc inexact d'insinuer qu'une « épistémologie locale afro » a pu rester étrangère à ces processus, surtout dans des zones ou des régions suffisamment bien reliées et en contact avec un monde social plus vaste.

Nous savons que les chercheurs de l'« afro-américanité » en sont conscients, et si dans leurs discours ils omettent ou minimisent l'importance de cet élément, c'est parce qu'ainsi ils peuvent participer à la narration de l' « essentialisme stratégique », mentionné plus haut. Cet « essentialisme » nécessite précisément une sélection d’informations

9 Jaime Arocha, cité par Restrepo, 2005, p.83.

10 À propos de ce village, le seul sur la côte caraïbe colombienne de population noire descendant de palenqueros (Noirs qui s'étaient échappés des plantations ou des maisons des colons, à l'instar des « Marrons »), Akomo Zoghe (2011) soutient que bien qu'existent des éléments de coïncidence linguistique et culturelle entre ce village et l'Afrique centrale, il est inapproprié, ou du moins exagéré, d'étendre l' « afro-palenquérité » à l'ensemble de la population noire du nord de la Colombie. 
et de données sur lesquelles va être fondée la théorie d'un « héritage africain $»$.

Ainsi, les documents ou les preuves sélectionnés, systématisés et divulgués obéissent à cette logique de création d'une identité ethnoculturelle « afro» qui soit opérationnelle. En ce sens, ce qui est pour nous valide au sujet du travail d'élaboration de la mémoire historiographique colombienne au XIXème siècle (Montes, 2009, 2015) l'est aussi pour l'historiographie, l'anthropologie et la sociologie qui se consacrent à la défense d'une « afro-colombianité ». Pour ces Sciences Sociales, comme pour de nombreuses autres, philosophie comprise, la sélection des données documentaires et empiriques peut finir par être motivée par un militantisme qui se confond avec l'objectivité scientifique. Ainsi, paradoxalement, une conséquence de ce que nous venons d'exposer s'observe dans le fait que, pour alimenter la narration de l'« afrodescendance », on a constamment recours à l'histoire de la colonialité. On revisite des conceptualisations telles qu'oppresseur/ opprimé, maître/esclave, colonisateur/colonisé, victime/bourreau, etc., qui au lieu d'être dépassées comme catégories explicatives, deviennent la base explicative pour le processus qui est supposé rompre avec ces conceptualisations $^{11}$. Ainsi, l'intention de rupture conceptuelle ne se concrétise pas ou se concrétise à moitié.

En ce qui concerne la stratégie narrative, à partir des éléments théoriques et conceptuels précédemment cités, s'établissent des relations de qualification/disqualification associées à l'idée d'une menace représentée par l'extériorité non « afro » et en conséquence

11 Ce soupçon est partagé par l'anthropologue colombien : "On pourrait arriver à mettre en évidence, ainsi, une hypothétique existence de continuités avec des formations discursives coloniales et racistes là où on se réclame d'une critique radicale de ces dernières. Ou bien, au contraire, on pourrait arriver à trouver que des objets, des concepts et des énoncés qui en surface semblaient former une unité sur laquelle s'était édifiée une narration du legs africain, ne forment pas d'unité mais qu'il existe des ruptures significatives du fait qu’ils répondent à différentes formations discursives » (Restrepo, 2005, p.92). 
nécessairement euro-centrée et ennemie. les défenseurs de l'« afrocolombianité » ont l'habitude de caricaturer leurs contradicteurs, en les accusant de mauvaise foi, d'être des alliés de l'« invisibilisation » du Noir. En somme, ces derniers sont vus comme des êtres « réprouvables éthiquement et politiquement $»$ :

D’un côté, il y a l'ignorance du pont Afrique-Amérique, l'eurocentrisme, la négation de l'ethnicité des afroaméricains, l'apologie du métissage, la négation de l'ancestralité et du legs africain, la datation de la créativité culturelle des afro-colombiens à partir de la déclaration juridique de la liberté, un cadre réactionnaire, les domaines carrés de la rigueur scientifique métropolitaine et un a-historicisme light et postmoderne, entre autres. De l'autre côté, la reconnaissance accomplie du pont AfriqueAmérique, le rejet de l'eurocentrisme, le dialogue libérateur Sud-Sud, la définition correcte de l'ethnicité au-delà de l'indigène qui inclut l'ancestralité afro-colombienne, la mise en question du métissage invisibilisant du legs africain, l'option éthique et politique émancipatrice des positions réactionnaires, et l'analyse scientifique dans sa vaste dimension historique et comparative. (Restrepo, 2005, p.105-106).

Nous partageons avec Restrepo l'idée que quand on met en question, ou qu'on examine avec le doute du chercheur, le discours de l' « afro-colombianité », on peut se faire accuser d'eurocentrisme imitateur et de négationnisme historique du « génocide » de la traite des Noirs et d'une « afro-genèse » évidente ; être vu comme un sorte d'agent epistémique au service des oppresseurs, dont la mission serait de perturber le processus libérateur des subalternisés historiques. Le retranchement théorique et discursif, la prise de position pour ou 
contre, dans le cadre d'une dichotomie du blanc et du noir, emblerait ainsi inéluctable.

Cette opposition devient évidente quand on aborde la relation entre connaissance et politique. Ainsi, pour certains défenseurs de l' « afro américanité », la critique des processus d'ethnisation des groupes subalternes noirs ne contribue qu'à détruire la progression vers la libération et l'empowerment de ces groupes. Face à eux se trouvent des auteurs pour qui la rigueur conceptuelle, et la recherche de connaissance pour elle-même, ne doivent pas être assujetties à la contingence du monde social ou des intérêts politiques, même si ceux-ci sont désirables. La dispute entre pouvoir et savoir évoquée par Foucault (1969) se dessine en arrière-plan de ces divergences.

Précisément, pour essayer de « sortir de la tranchée », il n'est pas sain de centrer l'« identité ethno-raciale » noire sur l'idée de ce que toute représentation sociale et historique d'un sujet détermine, nécessairement et durablement, sa position sociale. Ainsi, il convient de rompre avec cette prétendue relation de nécessité entre représentation historique du Noir opprimé et position sociale du sujet noir, afin de ne pas pérenniser l'usage stratégique de l' « essentialisme identitaire afro », qui bien qu'il ait été efficace, peut finir par se transformer en un nouveau métarécit, retardant des changements structurels et positifs, non seulement pour un groupe qui se cherche dans son identité ethnoculturelle et ethnoraciale, mais aussi pour l'ensemble de la société.

Nous connaissons l'existence de discours académiques qui répondent, de façon énergique, à cette manière de présenter la critique, en arguant qu'il s'agit d'une position « postmoderne » et constructiviste eurocentrée, qui cherche, d'une part, à fixer la notion de culture comme quelque chose de fabriqué, comme « un objet que l'acteur possède et non comme constitutif de la personne»(Mosquera, 2007, p. 218), et, par ailleurs, qui omet un facteur fondamental, le fait que la subalternisation 
des groupes ethno-raciaux s'inscrit dans une logique plus large et transnationale de « colonialité du pouvoir », puisque

En ce qui concerne les Noirs, afro-colombiens et raizales ${ }^{12}$, il existe une forte résistance à parler des relations de pouvoir existantes quant à la négation de l'égalité des chances pour ce groupe subalternisé et une grande ouverture et beaucoup de soutien à tout ce qui signifie un patrimoine immatériel, représenté dans des actes folkloriques, musicaux, gastronomiques et sportifs, sophisme de distraction dans lequel sont tombés une bonne partie des groupes qui constituent le Mouvement Social Afro-colombien (Mosquera, 2007, p. 219).

Nous partageons partiellement ces considérations, car avec l'accusation de "postmodernisme » eurocentré, s'alimente une théorie du grand complot théorique de l'hémisphère occidental, dans lequel se situeraient, dans une relation d'opposition, ceux qui sont capables de voir la vérité et les autres, encore dans la « servitude volontaire »du savoir. Nous ne pouvons pas nous étendre ici sur cette accusation assez discutable, qui se comprend seulement dans le cadre d'une dynamique d'intellectuel-militant, respectable, mais pas pour autant partagée. En revanche, nous sommes d'accord avec l'idée que, en Colombie, la différence culturelle et ethno-raciale est très focalisée sur les aspects de folklore et de patrimoine immatériel, qui finissent par créer un discours de « marché culturel $»^{13}$ et dépouillent le débat de sa substance théorique et normative, dans le sens philosophique.

12 Le terme raizal désigne, à partir de la CPC-91, les habitants des îles de l'archipel caribéen de San Andres, Providencia et Santa Catalina.

13 En Colombie, le nombre de dates et d'événements commémorant la présence « afro-colombienne », son histoire et des expressions est en constante augmentation depuis deux décennies. Ce choix, non critiquable en soi, favorise mallheureusement la « folklorisation de la différence culturelle ». Parmi ces différentes dates, on peut citer le « jour de l'afro-colombianité » (21 mai), les carnavals des chapitres noirs de Getsemani à Carthagène (en novembre), le festival de la musique, de la danse et la gastronomie afros ( Festival du Grand Hamac »), etc. 
A manière de conclusion et dans le même ordre d'idées, nous pouvons dire que ce n'est pas la reconnaissance de la diversité ethnoculturelle ou l'intégration à la vie politique et culturelle du sujet noir qui représente, selon nous, une menace - bien au contraire-, mais le fait que lesdits processus laissent de côté le conflit comme catégorie d'analyse et, en adoptant un discours de solidarité entre vaincus et subalternes, laissent entendre que toute tentative de dialogue est une menace contre les populations noires ou que les mesures d'affirmative action ne sont ni critiquables, ni négociables, puisqu'elles sont considérées comme le paiement d'une dette ancestrale et un « juste retour des choses $»$.

Une batterie des questionnements restent donc ouverte : pouvonsnous dire qu'avec l'« afro-colombianité » nous sommes devant un processus dans lequel, par le biais du discours du multiculturalisme politique (qui, rappelons-le, nous arrive depuis le haut car il est le résultat d'un changement constitutionnel), est récupérée une identité « afro » perdue et réduite au silence ? Ou plutôt : sommes-nous devant un phénomène de captation, de formation et de constitution de la condition « afro »? Si les réponses sont affirmatives, cela supposeraitil que les sujets identifiés comme «Afros » apprennent à ressentir une condition d'exclusion et de domination ethnoculturelle, dont ils n'étaient initialement pas porteurs, ni implicitement ni explicitement? Ainsi, l'« Afro » sert-il, surtout, à alimenter un discours idéologique de l'opprimé ?

\section{Références}

Araya Sandra. (2002). Las representaciones sociales: ejes teóricos para su discusión, in Cuadernos de Ciencias Sociales Facultad Latinoamericana de Ciencias Sociales (FLACSO). Costa Rica, FLACSO.

Berger Peter \& Luckann Thomas. (1991). La construcción social de la realidad. Buenos Aires, Amorrortu.

Akomo Zoghe Cyriaque. (2011). L'évangélisation des esclaves bantous et les résistances en Colombie de 1602 à 1774 . Thèse de doctorat soutenue le 
20/09, Université Paris 8, sous la direction de Jean-Pierre DuteilFoucault Michel. (1969). Larchéologie du savoir. Paris, Gallimard.

Foucault Michel. (2008). Le gouvernement de soi et des autres. Cours au Collège de France 1983. Paris, Seuil/Gallimard/Hautes études

Friedemann Nina. (1984). «Estudios de negros en la antropología colombiana: presencia e invisibilidad », in Arocha Jaime et Friedemann Nina (dir.), Un siglo de investigación social: antropología en Colombia. Bogotá, Etno. p. 507-572.

Friedemann Nina. (1992). «Negro en Colombia: Identidad e invisibilidad », in América Negra n³. Bogotá, Universidad Javeriana. p. 11-21.

Grueso Delfin. (2009). «Identidades étnicas, justicia y política transformativa », in Castellano G., Grueso D., Rodríguez M. (dir.). Identidad, Cultura y Política: perspectivas conceptuales, miradas empíricas. Cali, Universidad del Valle (coll. Libros de Investigación). p. 283-308.

Hernández Valeria. (2009). «Raza, cultura y comunicación: un lindo escándalo Lévi-straussiano », in Bilbao A., Gras S. \& Vermeren P. (dir.). Claude Lévi-Strauss en el pensamiento contemporáneo. Buenos Aires, Colihue. p. 89-103.

Lévi-Strauss Claude. (1987). Race et Histoire. Paris, Folio. (1ère éd. 1952, Unesco).

Mignolo Walter. (1996). « Herencias coloniales y teorías postcoloniales », in Gonzáles Stephan, Cultura y Tercer Mundo: 1. Cambios en el Saber Académico, Chap. IV. Venezuela, Nueva Sociedad. p. 99-136. Disponible en ligne :

www.cholonautas.edu.pe / Biblioteca Virtual de Ciencias Sociales.

Mignolo Walter. (2001). «Géopolitique de la connaissance : colonialité du pouvoir et différence », in Multitudes, n6, mars. p. 56-71.

Montes Angélica. (2009). «Ethnies multiples nation unique : Multiplicité ethnique et unité nationale, le cas colombien », in Revista Criterios, Universidad San Buenaventura Vol. 2, n¹.

Montes Montoya Angélica (2015), Représentation du sujet noir dans l'historiographie colombienne : le cas de Carthagène des Indes (18111815), Paris, l'Harmattan

Montes Montoya Angélica (2011), «Une construction de l'« être noir » : à partir d'une vision historiographique colombienne et d'un moment phénoménologique en France », coécrit avec Pauline Vermeren, in Éric Agbessi (dir.), Le Noir, couleur dangereuse ou transgressive?, Tome 1, Editions Le Manuscrit, Paris, p. 173 - 212. 
Moscovici Serge. (1989). Des représentations collectives aux représentations sociale : éléments pour une histoire, in Jodelet Denise (éd.), Les Représentations Sociales. Paris, PUF.

Quijano Anibal. (2000). « Colonialidad del poder, eurocentrismo y America Latina », in Lander Edgardo (dir.), La colonialidad del saber: Eurocentrismo y ciencias sociales. Caracas, IESALC-FACES/UCV.

Mosquera Claudia. (2007). « Reparaciones para negros, afrocolombianas y raizales como rescatados de la Trata Negrera Transatlántica y desterrados de la guerra en Colombia », in Mosquera Claudia \& Barcelo Luis Carlos (éd.), Afro-reparaciones: memorias de esclavitud y justicia reparativa para negros, afrocolombianas y raizales. Bogotá, Universidad Nacional de Colombia, CES.

Spivak Gayatri Chakravorty. (1987). In Other Worlds: Essays in Cultural Politics. Etats-Unis, Routledge. Traduit en français sous le titre En d'autres mondes, en d'autres mots. Essais de politique culturelle (2009, Payot).

Restrepo Eduardo. (2005). Política de las teorías y dilemas en los estudios de las colombias negras. Bogotá, Ediciones Universidad del Cauca.

Taylor Charles. (2004). Imaginarios sociales modernos. Barcelona, Paidos.

Akomo Zoghe Cyriaque. (2011). Lévangélisation des esclaves bantous et les résistances en Colombie de 1602 à 1774. 